Geometry $\&$ Topology

Volume 9 (2005) 2395-2415

Erratum 1

Published: 9 February 2005

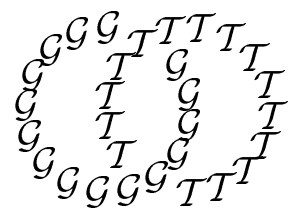

\title{
Correction to: Construction of 2-local finite groups of a type studied by Solomon and Benson
}

\author{
RAN LEVI \\ Bob Oliver \\ Department of Mathematical Sciences, University of Aberdeen \\ Meston Building 339, Aberdeen AB24 3UE, UK \\ and \\ LAGA, Institut Galilée, Av. J-B Clément \\ 93430 Villetaneuse, France \\ Email: ran@maths.abdn.ac.uk and bob@math.univ-paris13.fr
}

\begin{abstract}
A $p$-local finite group is an algebraic structure with a classifying space which has many of the properties of $p$-completed classifying spaces of finite groups. In our paper 2], we constructed a family of 2-local finite groups which are "exotic" in the following sense: they are based on certain fusion systems over the Sylow 2-subgroup of $\operatorname{Spin}_{7}(q)$ ( $q$ an odd prime power) shown by Solomon not to occur as the 2 -fusion in any actual finite group. As predicted by Benson, the classifying spaces of these 2-local finite groups are very closely related to the Dwyer-Wilkerson space $B D I(4)$. An error in our paper 2] was pointed out to us by Andy Chermak, and we correct that error here.
\end{abstract}

Keywords Classifying space, $p$-completion, finite groups, fusion

AMS Classification 55R35; 55R37, 20D06, 20D20

A saturated fusion system over a finite $p$-group $S$ is a category whose objects are the subgroups of $S$, whose morphisms are all monomorphisms between the subgroups, and which satisfy certain axioms first formulated by Puig, and also described at the start of the first section in [2]. The main result of [2] is the construction of saturated fusion systems over certain 2-groups, motivated by a theorem of Solomon [3, which implies that these systems cannot be induced by fusion in any finite group. Recently, Andy Chermak has pointed out to us that the fusion systems actually constructed in [2] are not saturated (do not satisfy all of Puig's axioms). In this note, we describe how to modify that 
construction in a way so as to obtain saturated fusion systems of the desired type, and explain why all of the results in [2] (aside from [2, Lemma A.10]) are true under this new construction.

The following is the main theorem in 2]:

Theorem 1.1 2, Theorem 2.1] Let $q$ be an odd prime power, and fix $S \in$ $\operatorname{Syl}_{2}\left(\operatorname{Spin}_{7}(q)\right)$. Let $z \in Z\left(\operatorname{Spin}_{7}(q)\right)$ be the central element of order 2. Then there is a saturated fusion system $\mathcal{F}=\mathcal{F}_{\text {Sol }}(q)$ which satisfies the following conditions:

(a) $C_{\mathcal{F}}(z)=\mathcal{F}_{S}\left(\operatorname{Spin}_{7}(q)\right)$ as fusion systems over $S$.

(b) All involutions of $S$ are $\mathcal{F}$-conjugate.

Furthermore, there is a unique centric linking system $\mathcal{L}=\mathcal{L}_{\text {Sol }}^{c}(q)$ associated to $\mathcal{F}$.

We have, in fact, found two errors in our proof of this theorem which we correct here. The more serious one is in [2, Lemma A.10], which is not true as stated: the last sentence in its proof is wrong. This has several implications on the rest of our construction, all of which are systematically treated here. There is also an error in the statement of [2, Lemma 2.8(b)] which is corrected below (Lemma 1.8).

We first state and prove here a corrected version of [2, Lemma A.10], and then state a modified version of the main technical proposition, 2, Proposition 1.2], used to prove saturation. Afterwards, we describe the changes which are needed in 2, Section 2] to prove the main theorem. In table 11 we list the correspondence between results and proofs in [2, Section 2] and those here. This is intended as a guide to the reader who is not yet familiar with [2], and who wants to read it simultaneously with this correction.

The only difference between 2, Lemma A.10] and the corrected version shown here is that in [2], we claimed that the "correction factor" $Z$ must lie in a certain subgroup of order 2 in $S L_{3}\left(\mathbb{Z} / 2^{k}\right)$, which is definitely not the case. Also, for convenience, we state this lemma here for matrices over the 2-adic integers $\widehat{\mathbb{Z}}_{2}$, instead of for matrices over the finite rings $\mathbb{Z} / 2^{k}$.

Lemma 1.2 (Modified [2, Lemma A.10]) Let $T_{1}$ and $T_{2}$ be the two maximal parabolic subgroups of $G L_{3}(2)$ :

$$
T_{1}=G L_{2}^{1}(\mathbb{Z} / 2)=\left\{\left(a_{i j}\right) \in G L_{3}(2) \mid a_{21}=a_{31}=0\right\}
$$




\begin{tabular}{|c|c|c|}
\hline Reference in [2] & Reference here & Remarks \\
\hline Proposition 1.2 & Proposition 1.3 & more general statement \\
\hline Theorem 2.1 & Theorem 1.1 & unchanged \\
\hline Definition 2.2, Lemma 2.3 & - & unchanged \\
\hline Definition 2.4 & - & omit definition of $\Gamma_{n}$ \\
\hline Prp. 2.5, Def. 2.6, Lem. 2.7 & - & unchanged \\
\hline- & Lem. 1.4, Prp. 1.5 & added \\
\hline- & Definition [1.6 & new definition of $\Gamma_{n}$ \\
\hline - & Lemma 1.7 & added \\
\hline Lemma 2.8 & Lemma 1.8 & $\begin{array}{l}\text { (b) restated, } \\
\text { new proofs of (b), (e) }\end{array}$ \\
\hline Proposition 2.9 & Proposition 1.9 & partly new proof \\
\hline Lemma 2.10 & Lemma 1.10 & partly new proof \\
\hline Proposition 2.11 & Proposition 1.11 & partly new proof \\
\hline
\end{tabular}

Table 1

and

$$
T_{2}=G L_{1}^{2}(\mathbb{Z} / 2)=\left\{\left(a_{i j}\right) \in G L_{3}(2) \mid a_{31}=a_{32}=0\right\} .
$$

Set $T_{0}=T_{1} \cap T_{2}$ : the group of upper triangular matrices in $G L_{3}(2)$. Assume, for some $k \geq 2$, that

$$
\mu_{i}: T_{i} \longrightarrow S L_{3}\left(\widehat{\mathbb{Z}}_{2}\right)
$$

are lifts of the inclusions $T_{i} \longrightarrow G L_{3}(2)=S L_{3}(2)$ (for $i=1,2$ ) such that $\left.\mu_{1}\right|_{T_{0}}=\left.\mu_{2}\right|_{T_{0}}$. Then there is a homomorphism

$$
\mu: G L_{3}(2) \longrightarrow S L_{3}\left(\widehat{\mathbb{Z}}_{2}\right),
$$

and an element $Z \in C_{S L_{3}\left(\widehat{\mathbb{Z}}_{2}\right)}\left(\mu_{1}\left(T_{0}\right)\right)$, such that $\left.\mu\right|_{T_{1}}=\mu_{1}$, and $\left.\mu\right|_{T_{2}}=c_{Z} \circ \mu_{2}$.

Proof By [1, Lemma 4.4], there is a lifting $\mu^{\prime}: G L_{3}(2) \longrightarrow S L_{3}\left(\widehat{\mathbb{Z}}_{2}\right)$ of the identity on $G L_{3}(2)$; and any two liftings to $S L_{3}\left(\widehat{\mathbb{Z}}_{2}\right)$ of the inclusion of $T_{1}$ or of $T_{2}$ into $G L_{3}(2)$ differ by conjugation by an element of $S L_{3}\left(\widehat{\mathbb{Z}}_{2}\right)$. In particular, there are elements $Z_{1}, Z_{2} \in S L_{3}\left(\widehat{\mathbb{Z}}_{2}\right)$ such that $\mu_{i}=\left.c_{Z_{i}} \circ \mu^{\prime}\right|_{T_{i}}($ for $i=1,2)$. Set $\mu=c_{Z_{1}} \circ \mu^{\prime}$, and $Z=Z_{1} Z_{2}^{-1}$. Then $\mu_{1}=\left.\mu\right|_{T_{i}}$, and $\left.\mu\right|_{T_{2}}=c_{Z} \circ \mu_{2}$. Since $\left.\mu_{1}\right|_{T_{0}}=\left.\mu_{2}\right|_{T_{0}}$, conjugation by $Z$ is the identity on $\mu_{1}\left(T_{0}\right)=\mu_{2}\left(T_{0}\right)$, and thus $Z \in C_{S L_{3}\left(\widehat{\mathbb{Z}}_{2}\right)}\left(\mu_{1}\left(T_{0}\right)\right)$.

Whenever $G$ is a finite group, $S \in \operatorname{Syl}_{p}(G), S_{0} \triangleleft S$, and $\Gamma \leq \operatorname{Aut}\left(S_{0}\right)$, then

$$
\left\langle\mathcal{F}_{S}(G) ; \mathcal{F}_{S_{0}}(\Gamma)\right\rangle
$$


denotes the smallest fusion system over $S$ which contains all $G$-fusion, and which also contains all restrictions of automorphisms in $\Gamma$. In other words, if $\mathcal{F}$ denotes this fusion system, then for each $P, Q \leq S, \operatorname{Hom}_{\mathcal{F}}(P, Q)$ is the set of all composites

$$
P=P_{0} \stackrel{\varphi_{1}}{\longrightarrow} P_{1} \stackrel{\varphi_{2}}{\longrightarrow} P_{2} \longrightarrow \cdots \longrightarrow P_{k-1} \stackrel{\varphi_{k}}{\longrightarrow} P_{k}=Q,
$$

where for each $i, P_{i} \leq S$, and either $\varphi_{i} \in \operatorname{Hom}_{G}\left(P_{i-1}, P_{i}\right)$, or (if $\left.P_{i-1}, P_{i} \leq S_{0}\right)$ $\varphi_{i}=\left.\psi_{i}\right|_{P_{i-1}}$ for some $\psi_{i} \in \Gamma$ such that $\psi_{i}\left(P_{i-1}\right)=P_{i}$.

Whenever $G$ is a finite group and $S \in \operatorname{Syl}_{p}(G)$, an automorphism $\varphi \in \operatorname{Aut}(S)$ is said to preserve $G$-fusion if for each $P, Q \leq S$ and each $\alpha \in \operatorname{Iso}(P, Q)$, $\alpha \in \operatorname{Iso}_{G}(P, Q)$ if and only if $\varphi \alpha \varphi^{-1} \in \operatorname{Iso}_{G}(\varphi(P), \varphi(Q))$. The proof of Theorem 1.1 is based on the following proposition.

Proposition 1.3 (Modified [2, Proposition 1.2]) Fix a finite group $G$, a prime $p$ dividing $|G|$, and a Sylow $p$-subgroup $S \in \operatorname{Syl}_{p}(G)$. Fix a normal subgroup $Z \triangleleft G$ of order $p$, an elementary abelian subgroup $U \triangleleft S$ of rank two containing $Z$ such that $C_{S}(U) \in \operatorname{Syl}_{p}\left(C_{G}(U)\right)$, and a group $\Gamma \leq \operatorname{Aut}\left(C_{S}(U)\right)$ of automorphisms which preserve all $C_{G}(U)$-fusion, and such that $\gamma(U)=U$ for all $\gamma \in \Gamma$. Set

$$
S_{0}=C_{S}(U) \quad \text { and } \quad \mathcal{F}=\left\langle\mathcal{F}_{S}(G) ; \mathcal{F}_{S_{0}}(\Gamma)\right\rangle,
$$

and assume the following hold.

(a) All subgroups of order $p$ in $S$ different from $Z$ are $G$-conjugate.

(b) $\Gamma$ permutes transitively the subgroups of order $p$ in $U$.

(c) $\{\varphi \in \Gamma \mid \varphi(Z)=Z\}=\operatorname{Aut}_{N_{G}(U)}\left(C_{S}(U)\right)$.

(d) For each $E \leq S$ which is elementary abelian of rank three, contains $U$, and is fully centralized in $\mathcal{F}_{S}(G)$,

$$
\left\{\alpha \in \operatorname{Aut}_{\mathcal{F}}\left(C_{S}(E)\right) \mid \alpha(Z)=Z\right\}=\operatorname{Aut}_{G}\left(C_{S}(E)\right) .
$$

(e) For all $E, E^{\prime} \leq S$ which are elementary abelian of rank three and contain $U$, if $E$ and $E^{\prime}$ are $\Gamma$-conjugate, then they are $G$-conjugate.

Then $\mathcal{F}$ is a saturated fusion system over $S$. Also, for any $P \leq S$ such that $Z \leq P$,

$$
\left\{\varphi \in \operatorname{Hom}_{\mathcal{F}}(P, S) \mid \varphi(Z)=Z\right\}=\operatorname{Hom}_{G}(P, S) .
$$


This proposition is slightly more general than [2, Proposition 1.2], in that $\Gamma$ is assumed only to be a group of automorphisms of $C_{S}(U)$ which preserves $C_{G}(U)$-fusion, and not a group of automorphisms of $C_{G}(U)$ itself. This extra generality is necessary when proving that the fusion systems $\mathcal{F}_{\text {Sol }}(q)$, under our modified construction, are saturated. The changes needed to prove this more general version of [2, Proposition 1.2] are described in Section 2.

Proposition 1.3 is applied with $G=\operatorname{Spin}_{7}(q), Z=Z(G)$, and $S \in \operatorname{Syl}_{2}(G)$, $U \leq S$, and $\Gamma \leq \operatorname{Aut}\left(C_{G}(U)\right)$ to be chosen shortly. The error in [2] arose in the choice of $\Gamma$, as will be explained in detail below.

We first recall some of the definitions and notation used in 2]. Throughout, we fix an odd prime power $q$, let $\mathbb{F}_{q}$ be a field with $q$ elements, and let $\overline{\mathbb{F}}_{q}$ be its algebraic closure. We write $S L_{2}\left(q^{\infty}\right)=S L_{2}\left(\overline{\mathbb{F}}_{q}\right), \operatorname{Spin}_{7}\left(q^{\infty}\right)=\operatorname{Spin}_{7}\left(\overline{\mathbb{F}}_{q}\right)$, etc., for short. For each $n, \psi^{q^{n}}$ denotes the automorphism of $\operatorname{Spin}_{7}\left(q^{\infty}\right)$ or of $S L_{2}\left(q^{\infty}\right)$ induced by the field isomorphism $\left(x \mapsto x^{q^{n}}\right)$. We then fix elements $z, z_{1} \in \operatorname{Spin}_{7}(q)$ of order 2 , where $\langle z\rangle=Z\left(\operatorname{Spin}_{7}(q)\right)$, set $U=\left\langle z, z_{1}\right\rangle$, and construct an explicit homomorphism

$$
\omega: S L_{2}\left(q^{\infty}\right)^{3} \longrightarrow \operatorname{Spin}_{7}\left(q^{\infty}\right)
$$

such that

$$
\operatorname{Im}(\omega)=C_{\operatorname{Spin}_{7}\left(q^{\infty}\right)}(U) \quad \text { and } \quad \operatorname{Ker}(\omega)=\langle(-I,-I,-I)\rangle .
$$

Write

$H\left(q^{\infty}\right) \stackrel{\text { def }}{=} \omega\left(S L_{2}\left(q^{\infty}\right)^{3}\right)=C_{\operatorname{Spin}_{7}\left(q^{\infty}\right)}(U) \quad$ and $\quad \llbracket X_{1}, X_{2}, X_{3} \rrbracket=\omega\left(X_{1}, X_{2}, X_{3}\right)$

for short. In particular,

$$
z=\llbracket I, I,-I \rrbracket \quad \text { and } \quad z_{1}=\llbracket-I, I, I \rrbracket,
$$

and thus

$$
U=\{\llbracket \pm I, \pm I, \pm I \rrbracket\}
$$

(with all combinations of signs). By [2, Lemma $2.3 \&$ Proposition 2.5], there is an element $\tau \in N_{\operatorname{Spin}_{7}(q)}(U)$ of order 2 such that

$$
\tau \cdot \llbracket X_{1}, X_{2}, X_{3} \rrbracket \cdot \tau^{-1}=\llbracket X_{2}, X_{1}, X_{3} \rrbracket
$$

for all $X_{1}, X_{2}, X_{3} \in S L_{2}\left(q^{\infty}\right)$, and such that

$$
N_{\operatorname{Spin}_{7}\left(q^{\infty}\right)}(U)=H\left(q^{\infty}\right) \cdot\langle\tau\rangle .
$$

We next fix elements $A, B \in S L_{2}(q)$ of order 4 , such that $\langle A, B\rangle \cong Q_{8}$ (a quaternion group of order 8). Most of the following notation is taken from [2, Definition 2.6]. We set

$$
\widehat{A}=\llbracket A, A, A \rrbracket \quad \text { and } \quad \widehat{B}=\llbracket B, B, B \rrbracket ;
$$




$$
C\left(q^{\infty}\right)=\left\{X \in C_{S L_{2}\left(q^{\infty}\right)}(A) \mid X^{2^{k}}=I, \text { some } k\right\} \cong \mathbb{Z} / 2^{\infty}
$$

and

$$
Q\left(q^{\infty}\right)=\left\langle C\left(q^{\infty}\right), B\right\rangle .
$$

Here, $\mathbb{Z} / 2^{\infty}$ means a union of cyclic 2 -groups $\mathbb{Z} / 2^{n}$ for all $n$; equivalently, the group $\mathbb{Z}\left[\frac{1}{2}\right] / \mathbb{Z}$. We then define

$$
\begin{aligned}
A\left(q^{\infty}\right) & =\omega\left(C\left(q^{\infty}\right)^{3}\right) \cong\left(\mathbb{Z} / 2^{\infty}\right)^{3}, \\
S_{0}\left(q^{\infty}\right) & =\omega\left(Q\left(q^{\infty}\right)^{3}\right) \leq H\left(q^{\infty}\right) \\
S\left(q^{\infty}\right) & =S_{0}\left(q^{\infty}\right) \cdot\langle\tau\rangle \leq H\left(q^{\infty}\right) \cdot\langle\tau\rangle \leq \operatorname{Spin}_{7}\left(q^{\infty}\right) .
\end{aligned}
$$

In all cases, whenever a subgroup $\Theta\left(q^{\infty}\right) \leq \operatorname{Spin}_{7}\left(q^{\infty}\right)$ has been defined, we set

$$
\Theta\left(q^{n}\right)=\Theta\left(q^{\infty}\right) \cap \operatorname{Spin}_{7}\left(q^{n}\right) .
$$

Since $\operatorname{Spin}_{7}\left(q^{n}\right)$ is the fixed subgroup of $\psi^{q^{n}}$ acting on $\operatorname{Spin}_{7}\left(q^{\infty}\right)$ (cf. 2, Lemma A.3]), $H\left(q^{n}\right) \leq H\left(q^{\infty}\right)$ is the subgroup of all elements of the form $\llbracket X_{1}, X_{2}, X_{3} \rrbracket$, where either $X_{i} \in S L_{2}\left(q^{n}\right)$ for each $i$, or $\psi^{q^{n}}\left(X_{i}\right)=-X_{i}$ for each $i$. By [2, Lemma 2.7], for all $n$,

$$
S\left(q^{n}\right) \in \operatorname{Syl}_{2}\left(\operatorname{Spin}_{7}\left(q^{n}\right)\right) .
$$

The following lemma is what is needed to tell us how to choose a subgroup $\Gamma_{n} \leq \operatorname{Aut}\left(S_{0}\left(q^{n}\right)\right)$ so that the fusion system $\left\langle\mathcal{F}_{S\left(q^{n}\right)}\left(\operatorname{Spin}_{7}\left(q^{n}\right)\right) ; \mathcal{F}_{S_{0}\left(q^{n}\right)}\left(\Gamma_{n}\right)\right\rangle$ is saturated. Note that since each element of $C\left(q^{\infty}\right)$ has 2-power order, it makes sense to write $X^{u} \in C\left(q^{\infty}\right)$ for $X \in C\left(q^{\infty}\right)$ and $u \in \widehat{\mathbb{Z}}_{2}$.

Lemma 1.4 Assume $\alpha \in \operatorname{Aut}\left(A\left(q^{\infty}\right)\right)$ centralizes $\operatorname{Aut}_{S\left(q^{\infty}\right)}\left(A\left(q^{\infty}\right)\right)$. Then $\alpha$ has the form

$$
\alpha\left(\llbracket X_{1}, X_{2}, X_{3} \rrbracket\right)=\llbracket X_{1}^{v}, X_{2}^{v}, X_{3}^{u} \rrbracket
$$

for some $u, v \in\left(\widehat{\mathbb{Z}}_{2}\right)^{*}$.

\section{Proof Set}

$$
\Delta_{0}=\operatorname{Aut}_{S\left(q^{\infty}\right)}\left(A\left(q^{\infty}\right)\right)=\left\langle c_{\llbracket B, I, I \rrbracket}, c_{\llbracket I, B, I \rrbracket}, c_{\llbracket I, I, B \rrbracket}, c_{\tau}\right\rangle
$$

for short. The second equality follows since $S\left(q^{\infty}\right)$ is by definition generated by $A\left(q^{\infty}\right)$ and the four elements listed. Set

$$
A_{1} \stackrel{\text { def }}{=}\left\langle z, z_{1}, \widehat{A}\right\rangle \cong C_{2}^{3}
$$

the 2-torsion subgroup in $A\left(q^{\infty}\right)$. The image of $\Delta_{0} \leq \operatorname{Aut}\left(A\left(q^{\infty}\right)\right)$ in the group $\operatorname{Aut}\left(A_{1}\right) \cong G L_{3}(2)$ (the image under restriction) is the group of all 
automorphisms which leave $\langle z\rangle$ and $U=\left\langle z, z_{1}\right\rangle$ invariant (ie, the group of upper triangular matrices with respect to the ordered basis $\left.\left\{z, z_{1}, \widehat{A}\right\}\right)$.

By assumption, $\left[\alpha, \Delta_{0}\right]=1$, and in particular, $\alpha \Delta_{0} \alpha^{-1}=\Delta_{0}$. Since each element of $\Delta_{0}$ sends $U$ to itself, this means that each element of $\Delta_{0}$ also sends $\alpha(U)$ to itself. Also, $U$ is the only subgroup of rank 2 left invariant by all elements of $\Delta_{0}$ (since $\Delta_{0}$ contains all automorphisms which leave $\langle z\rangle$ and $U$ invariant), and hence $\alpha(U)=U$.

It follows that $\alpha$ induces an automorphism $\alpha^{\prime}$ of $A\left(q^{\infty}\right) / U=\left(C\left(q^{\infty}\right) /\langle-I\rangle\right)^{3}$. Also, $\alpha^{\prime}$ commutes with the following automorphisms of $\left(C\left(q^{\infty}\right) /\langle-I\rangle\right)^{3}$ :

$$
\left(Y_{1}, Y_{2}, Y_{3}\right) \mapsto\left(Y_{1}^{ \pm 1}, Y_{2}^{ \pm 1}, Y_{3}^{ \pm 1}\right) \quad \text { and } \quad\left(Y_{1}, Y_{2}, Y_{3}\right) \mapsto\left(Y_{2}, Y_{1}, Y_{3}\right)
$$

since these are induced by automorphisms in $\Delta_{0}$. Hence $\alpha^{\prime}$ has the form $\alpha^{\prime}\left(Y_{1}, Y_{2}, Y_{3}\right)=\left(Y_{1}^{v}, Y_{2}^{v}, Y_{3}^{u}\right)$ for some $u, v \in\left(\widehat{\mathbb{Z}}_{2}\right)^{*}$. Thus for all $X_{1}, X_{2}, X_{3} \in$ $C\left(q^{\infty}\right)$

$$
\alpha\left(\llbracket X_{1}, X_{2}, X_{3} \rrbracket\right)=\llbracket \pm X_{1}^{v}, \pm X_{2}^{v}, \pm X_{3}^{u} \rrbracket .
$$

Since all elements of $C\left(q^{\infty}\right)$ are squares, these signs must all be positive, and $\alpha$ has the form $\alpha\left(\llbracket X_{1}, X_{2}, X_{3} \rrbracket=\llbracket X_{1}^{v}, X_{2}^{v}, X_{3}^{u} \rrbracket\right.$.

For each $u \in\left(\widehat{\mathbb{Z}}_{2}\right)^{*}$, let $\delta_{u} \in \operatorname{Aut}\left(A\left(q^{\infty}\right)\right)$ be the automorphism

$$
\delta_{u}\left(\llbracket X_{1}, X_{2}, X_{3} \rrbracket\right)=\left[X_{1}, X_{2}, X_{3}^{u}\right] .
$$

Define $\gamma, \gamma_{u} \in \operatorname{Aut}\left(A\left(q^{\infty}\right)\right)$ by setting

$$
\gamma\left(\llbracket X_{1}, X_{2}, X_{3} \rrbracket\right)=\llbracket X_{3}, X_{1}, X_{2} \rrbracket \quad \text { and } \quad \gamma_{u}=\delta_{u} \gamma \delta_{u}^{-1} .
$$

Proposition 1.5 There is an element $u \in \widehat{\mathbb{Z}}_{2}$ such that $u \equiv 1(\bmod 4)$, and such that the subgroup $\Omega_{u} \leq \operatorname{Aut}\left(A\left(q^{\infty}\right)\right)$ given by

$$
\Omega_{u} \stackrel{\text { def }}{=}\left\langle\operatorname{Aut}_{\operatorname{Spin}_{7}\left(q^{\infty}\right)}\left(A\left(q^{\infty}\right)\right), \gamma_{u}\right\rangle
$$

is isomorphic to $C_{2} \times G L_{3}(2)$. Furthermore, the following hold:

(a) The subgroup of elements of $\Omega_{u}$ which act via the identity on all 2-torsion in $A\left(q^{\infty}\right)$ has order 2 , and contains only the identity and the automorphism $\left(g \mapsto g^{-1}\right)$.

(b) For each $n \geq 1$,

$$
\left\langle\operatorname{Aut}_{\operatorname{Spin}_{7}\left(q^{n}\right)}\left(A\left(q^{n}\right)\right), \gamma_{u}\right\rangle \cong C_{2} \times G L_{3}(2) .
$$


Proof For each $k \geq 1$, let $A_{k} \leq A\left(q^{\infty}\right)$ denote the $2^{k}$-torsion subgroup. In particular,

$$
A_{1}=\left\langle z, z_{1}, \widehat{A}\right\rangle \cong C_{2}^{3}, \quad \text { where } \quad \widehat{A}=\llbracket A, A, A \rrbracket .
$$

Let $R_{1}, R_{2}, \cdots \in C\left(q^{\infty}\right) \cong \mathbb{Z} / 2^{\infty}$ be elements such that $R_{1}=-I, R_{2}=A$, and $\left(R_{i}\right)^{2}=R_{i-1}$ for all $k \geq 2$. Thus, $\left|R_{i}\right|=2^{i}$ for all $i$. For each $k \geq 1$, let $\left\{\mathbf{r}_{1}^{(k)}, \mathbf{r}_{2}^{(k)}, \mathbf{r}_{3}^{(k)}\right\}$ be the basis of $A_{k}$ defined by

$$
\mathbf{r}_{1}^{(k)}=\llbracket I, I, R_{k} \rrbracket, \quad \mathbf{r}_{2}^{(k)}=\llbracket R_{k}, I, I \rrbracket, \quad \text { and } \quad \mathbf{r}_{3}^{(k)}=\llbracket R_{k+1}, R_{k+1}, R_{k+1} \rrbracket .
$$

In particular, $\mathbf{r}_{1}^{(1)}=z, \mathbf{r}_{2}^{(1)}=z_{1}$, and $\mathbf{r}_{3}^{(1)}=\widehat{A}$. Using these bases, we identify $\operatorname{Aut}\left(A_{k}\right)=G L_{3}\left(\mathbb{Z} / 2^{k}\right)$ and $\operatorname{Aut}\left(A\left(q^{\infty}\right)\right)=G L_{3}\left(\widehat{\mathbb{Z}}_{2}\right)$.

Set

$$
\Delta_{0}=\operatorname{Aut}_{S\left(q^{\infty}\right)}\left(A\left(q^{\infty}\right)\right), \quad \Delta_{1}=\operatorname{Aut}_{\operatorname{Spin}_{7}\left(q^{\infty}\right)}\left(A\left(q^{\infty}\right)\right), \quad \text { and } \quad \Delta_{2}=\left\langle\Delta_{0}, \gamma\right\rangle .
$$

In particular, $\Delta_{2}$ is the group of all signed permutations

$$
\llbracket X_{1}, X_{2}, X_{3} \rrbracket \mapsto \llbracket X_{\sigma(1)}^{ \pm 1}, X_{\sigma(2)}^{ \pm 1}, X_{\sigma(3)}^{ \pm 1} \rrbracket
$$

for $\sigma \in \Sigma_{3}$.

For each $i=0,1,2$ and each $k \geq 1$, let $\Delta_{i}^{(k)} \leq \operatorname{Aut}\left(A_{k}\right)$ be the image of $\Delta_{i}$ under restriction. By [2, Proposition A.8], $\Delta_{1}^{(1)}=\operatorname{Aut}_{\text {Spin }}\left(A_{1}\right)$ is the group of all elements of $\operatorname{Aut}\left(A_{1}\right) \cong G L_{3}(\mathbb{Z} / 2)$ which send $z$ to itself. Also, $\Delta_{0}^{(1)}$ was seen in the proof of Lemma 1.4 to be the group of all automorphisms of $A_{1}$ which leaves both $z$ and $U=\left\langle z, z_{1}\right\rangle$ invariant; and a similar argument shows that $\Delta_{2}^{(1)}$ is the group of all automorphisms of $A_{1}$ which leaves $U$ invariant. Hence, with respect to the ordered basis $\left\{z, z_{1}, \widehat{A}\right\}$ of $A_{1}$, each group $\Delta_{i}^{(1)} \leq \operatorname{Aut}\left(A_{1}\right)$ $(i=0,1,2)$ can be identified with the subgroup $T_{i} \leq G L_{3}(\mathbb{Z} / 2)$ of Lemma 1.2

By [2, Proposition 2.5],

$$
C_{\operatorname{Spin}_{7}\left(q^{\infty}\right)}(U)=H\left(q^{\infty}\right) \cong\left(S L_{2}\left(q^{\infty}\right)^{3}\right) /\langle(-I,-I,-I)\rangle .
$$

An element $\llbracket X_{1}, X_{2}, X_{3} \rrbracket \in H\left(q^{\infty}\right)\left(X_{i} \in S L_{2}\left(q^{\infty}\right)\right)$ centralizes $\widehat{A} \stackrel{\text { def }}{=} \llbracket A, A, A \rrbracket$ if and only if $\left[X_{i}, A\right]=1$ for each $i$, or $X_{i} A X_{i}^{-1}=-A=A^{-1}$ for each $i$. Set $\bar{C}=C_{S L_{2}\left(q^{\infty}\right)}(A)$. This is an abelian group (the union of the finite cyclic groups $\left.C_{S L_{2}\left(q^{n}\right)}(A)\right)$, and $N_{S L_{2}\left(q^{\infty}\right)}(A)=\bar{C} \cdot\langle B\rangle$. Hence, since $A_{1}=\langle U, \widehat{A}\rangle$ and $\widehat{B}=\llbracket B, B, B \rrbracket$, we have

$$
C_{\operatorname{Spin}_{7}\left(q^{\infty}\right)}\left(A_{1}\right)=C_{H\left(q^{\infty}\right)}(\widehat{A})=\omega\left(\bar{C}^{3}\right) \cdot\langle\widehat{B}\rangle .
$$


Since $\omega\left(\bar{C}^{3}\right)$ is abelian (thus centralizes $A\left(q^{\infty}\right)$ ), this shows that the kernel of each of the projection maps $\Delta_{i} \longrightarrow \Delta_{i}^{(1)}$ is generated by conjugation by $\widehat{B}$; ie, by the automorphism $\left(g \mapsto g^{-1}\right)$.

Since each $\Delta_{i}$ is finite, their elements all have determinant of finite order in $\left(\widehat{\mathbb{Z}}_{2}\right)^{*}$, hence are \pm 1 in all cases. Also, for each $i, \Delta_{i}$ surjects onto $\Delta_{i}^{(1)}=T_{i}$ with kernel generated by the automorphism $\left(g \mapsto g^{-1}\right)$ of determinant $(-1)$. Hence the elements of determinant one in $\Delta_{i}$ are sent isomorphically to $T_{i}$, and define a lift

$$
\mu_{i}: T_{i} \longrightarrow S L_{3}\left(\widehat{\mathbb{Z}}_{2}\right)
$$

with respect to the given bases. In particular, $\left.\mu_{1}\right|_{T_{0}}=\left.\mu_{2}\right|_{T_{0}}=\mu_{0}$.

For all $i, j, k \in\left(\widehat{\mathbb{Z}}_{2}\right)^{*}$, we define $\psi_{i, j, k} \in \operatorname{Aut}\left(A\left(q^{\infty}\right)\right)$ by setting

$$
\psi_{i, j, k}\left(\llbracket X_{1}, X_{2}, X_{3} \rrbracket\right)=\llbracket X_{1}^{i}, X_{2}^{j}, X_{3}^{k} \rrbracket
$$

for all $\llbracket X_{1}, X_{2}, X_{3} \rrbracket \in A\left(q^{\infty}\right)$. Recall that $c_{\tau}\left(\llbracket X_{1}, X_{2}, X_{3} \rrbracket\right)=\llbracket X_{2}, X_{1}, X_{3} \rrbracket$ (by choice of $\tau)$. Since $\Delta_{0}=\operatorname{Aut}_{S\left(q^{\infty}\right)}\left(A\left(q^{\infty}\right)\right)$ is generated by $c_{\llbracket B, I, I \rrbracket}, c_{\llbracket I, B, I \rrbracket}$, $c_{\llbracket I, I, B \rrbracket}$, and $c_{\tau}$ (corresponding to generators of $\left.S\left(q^{\infty}\right) / A\left(q^{\infty}\right)\right)$, $\mu_{0}$ has image

$$
\operatorname{Im}\left(\mu_{0}\right)=\mu_{1}\left(T_{0}\right)=\left\langle\psi_{-1,-1,1}, \psi_{1,-1,-1}, c_{\tau}\right\rangle,
$$

where $\psi_{-1,-1,1}=c_{\llbracket B, B, I \rrbracket}$ and $\psi_{1,-1,-1}=c_{\llbracket I, B, B \rrbracket}$.

By Lemma 1.2, there is a homomorphism

$$
\mu: G L_{3}(2) \longrightarrow S L_{3}\left(\widehat{\mathbb{Z}}_{2}\right),
$$

and an element $Z \in \operatorname{Aut}\left(A\left(q^{\infty}\right)\right) \cong G L_{3}\left(\widehat{\mathbb{Z}}_{2}\right)$ which commutes with all elements of $\mu_{1}\left(T_{0}\right)$, such that $\left.\mu\right|_{T_{1}}=\mu_{1}$ and $\left.\mu\right|_{T_{2}}=c_{Z} \circ \mu_{2}$. By Lemma 1.4 $Z=\psi_{v, v, u}$ (using the above notation) for some $u, v \in\left(\widehat{\mathbb{Z}}_{2}\right)^{*}$. Since $\psi_{v, v, v}$ lies in the center of $\operatorname{Aut}\left(A\left(q^{\infty}\right)\right)$ (it sends every element to its $v$-th power), we can assume that $v=1$ (without changing $c_{Z}$ ), and thus that $Z=\psi_{1,1, u}=\delta_{u}$. Finally, since $\delta_{-1}=\psi_{1,1,-1} \in \Delta_{0}$, we can replace $\delta_{u}$ by $\delta_{-u}$ if necessary, and assume that $u \equiv 1(\bmod 4)$.

Under the identification $G L_{3}\left(\widehat{\mathbb{Z}}_{2}\right)=\operatorname{Aut}\left(A\left(q^{\infty}\right)\right)$, we now have

$$
\operatorname{Aut}_{\operatorname{Spin}_{7}\left(q^{\infty}\right)}\left(A\left(q^{\infty}\right)\right)=\left(g \mapsto g^{-1}\right) \times \mu\left(T_{1}\right)
$$

and

$$
\gamma_{u}=\delta_{u} \gamma \delta_{u}^{-1}=c_{Z}(\gamma)=\mu\left(\left(\begin{array}{lll}
0 & 1 & 0 \\
1 & 1 & 0 \\
0 & 0 & 1
\end{array}\right)\right),
$$

where the matrix is that of $\left.\gamma\right|_{A_{1}}$ with respect to the basis $\left\{\mathbf{r}_{1}^{(1)}, \mathbf{r}_{2}^{(1)}, \mathbf{r}_{3}^{(1)}\right\}$. Also, $T_{1}$ is a maximal subgroup of $G L_{3}(2)$ - the subgroup of invertible matrices 
which send $\mathbf{r}_{1}^{(1)}$ to itself - and so $T_{1}$ together with this matrix generate $G L_{3}(2)$. Thus

$$
\Omega_{u}=\left\langle\operatorname{Aut}_{\operatorname{Spin}_{7}\left(q^{\infty}\right)}\left(A\left(q^{\infty}\right)\right), \gamma_{u}\right\rangle=\left\langle g \mapsto g^{-1}\right\rangle \times \mu\left(G L_{3}(2)\right) \cong C_{2} \times G L_{3}(2) .
$$

This proves the first claim in the proposition. Point (a) follows by construction, since each nonidentity element of $\mu\left(G L_{3}(2)\right)$ acts nontrivially on $A_{1}$. Point (b) follows from (2), once we know that each element of $\operatorname{Spin}_{7}\left(q^{n}\right)$ which normalizes $A_{1}$ (hence which normalizes $\left.A\left(q^{n}\right)\right)$ also normalizes $A\left(q^{\infty}\right)$ - and this follows from (1).

We are now in a position to define the fusion systems we want. Roughly, they are generated by the fusion systems of $\operatorname{Spin}_{7}\left(q^{n}\right)$ together with one extra automorphism: the cyclic permutation $\llbracket X_{1}, X_{2}, X_{3} \rrbracket \mapsto \llbracket X_{3}, X_{1}, X_{2} \rrbracket$ "twisted" by the automorphism $\delta_{u}$ of the last proposition. By comparison, the construction in 2 was similar but without the twisting (ie, done with $u=1$ ), and the resulting fusion system is, in fact, not saturated.

We regard $Q\left(q^{\infty}\right)=C\left(q^{\infty}\right) \rtimes\langle B\rangle$ as an infinite quaternion group: $B A^{\prime} B^{-1}=$ $A^{\prime-1}$ for each $A^{\prime} \in C\left(q^{\infty}\right)$, and each element of the coset $C\left(q^{\infty}\right) \cdot B$ has order 4. Hence any automorphism of $C\left(q^{\infty}\right)$ extends to a unique automorphism of $Q\left(q^{\infty}\right)$ which sends $B$ to itself.

Definition 1.6 Let $u \in\left(\widehat{\mathbb{Z}}_{2}\right)^{*}$ be as in Proposition 1.5, Let

$$
\widehat{\gamma}, \widehat{\delta}_{u} \in \operatorname{Aut}\left(S_{0}\left(q^{\infty}\right)\right)
$$

be the automorphisms

$$
\widehat{\gamma}\left(\llbracket X_{1}, X_{2}, X_{3} \rrbracket\right)=\llbracket X_{3}, X_{1}, X_{2} \rrbracket
$$

and

$$
\widehat{\delta}_{u}\left(\llbracket X_{1}, X_{2}, A^{\prime} B^{j} \rrbracket\right)=\llbracket X_{1}, X_{2},\left(A^{\prime}\right)^{u} B^{j} \rrbracket
$$

for all $X_{i} \in Q\left(q^{\infty}\right), A^{\prime} \in C\left(q^{\infty}\right)$, and $i, j \in \mathbb{Z}$; and set $\widehat{\gamma}_{u}=\widehat{\delta}_{u} \widehat{\gamma}_{u}^{-1} \in$ $\operatorname{Aut}\left(S_{0}\left(q^{\infty}\right)\right)$. For each $n \geq 1$, set

$$
\Gamma_{n}=\left\langle\operatorname{Inn}\left(S_{0}\left(q^{n}\right)\right), c_{\tau}, \widehat{\gamma}_{u}\right\rangle \leq \operatorname{Aut}\left(S_{0}\left(q^{n}\right)\right) ;
$$

and set

$$
\mathcal{F}_{n}=\mathcal{F}_{\text {Sol }}\left(q^{n}\right)=\left\langle\mathcal{F}_{S\left(q^{n}\right)}\left(\operatorname{Spin}_{7}\left(q^{n}\right)\right), \mathcal{F}_{S_{0}\left(q^{n}\right)}\left(\Gamma_{n}\right)\right\rangle
$$

In order to be able to apply Proposition [1.3, it is important to know that $\Gamma_{n}$ is fusion preserving. This follows immediately from the following lemma. 
Lemma 1.7 For each $n \geq 1$, the automorphisms $c_{\tau}, \widehat{\gamma}, \widehat{\delta}_{u}$, and $\widehat{\gamma}_{u}$ all preserve $H\left(q^{n}\right)$-fusion in $S_{0}\left(q^{n}\right)$.

Proof The fusion in $S L_{2}\left(q^{n}\right)$ is generated by inner automorphisms of its Sylow subgroup $Q\left(q^{n}\right)$, together with the groups $\operatorname{Aut}(P)$ for subgroups $P \leq Q\left(q^{n}\right)$ isomorphic to $Q_{8}$. This follows from Alperin's fusion theorem, since these are the only subgroups whose automorphism group is not a 2 -group. Thus any automorphism of $Q\left(q^{n}\right)$ - in particular, the automorphism $\varphi_{u}$ defined by $\varphi_{u}\left(A^{\prime}\right)=A^{\prime u}, \varphi_{u}(B)=B$ for $A^{\prime} \in C\left(q^{n}\right)$ - preserves fusion.

Set $H_{0}\left(q^{n}\right)=\left\{\llbracket X_{1}, X_{2}, X_{3} \rrbracket \mid X_{i} \in S L_{2}\left(q^{n}\right), \forall i\right\}$. Fix a generator $Y$ of $C\left(q^{2 n}\right)$; then $C\left(q^{n}\right)=\left\langle Y^{2}\right\rangle$, and $\psi^{q^{n}}(Y)=-Y$. For each $g=\llbracket X_{1}, X_{2}, X_{3} \rrbracket \in H\left(q^{n}\right)$, $\llbracket \psi^{q^{n}}\left(X_{1}\right), \psi^{q^{n}}\left(X_{2}\right), \psi^{q^{n}}\left(X_{3}\right) \rrbracket=\llbracket X_{1}, X_{2}, X_{3} \rrbracket ;$ and hence there is some fixed $\epsilon= \pm 1$ for which $\psi^{q^{n}}\left(X_{i}\right)=\epsilon \cdot X_{i}$ for $i=1,2,3$. When $\epsilon=1$, this means that $X_{i} \in S L_{2}\left(q^{n}\right)$ for each $i$; while if $\epsilon=-1$ it means that $X_{i} \in S L_{2}\left(q^{n}\right) \cdot Y$ for each $i$. So every element of $H\left(q^{n}\right)$ either lies in $H_{0}\left(q^{n}\right)$, or has the form $g \cdot \llbracket Y, Y, Y \rrbracket$ for some $g \in H_{0}\left(q^{n}\right)$.

Assume $g \in H\left(q^{n}\right)$ and $P, Q \leq S_{0}\left(q^{n}\right)$ are such that $g P g^{-1}=Q$. Clearly, $P \leq H_{0}\left(q^{n}\right)$ if and only if $Q \leq H_{0}\left(q^{n}\right)\left(H_{0}\left(q^{n}\right)\right.$ is normal in $\left.H\left(q^{n}\right)\right)$. We claim that there is $h \in H\left(q^{n}\right)$ such that

$$
h \widehat{\delta}_{u}(P) h^{-1}=\widehat{\delta}_{u}(Q) \quad \text { and }\left.\quad c_{h} \circ \widehat{\delta}_{u}\right|_{P}=\left.\widehat{\delta}_{u} \circ c_{g}\right|_{P} .
$$

Let $P_{i}, Q_{i} \leq S L_{2}\left(q^{2 n}\right)$ be the projections of $P$ and $Q$ to the $i$-th factor $(i=$ $1,2,3$ ), and write $g=\llbracket X_{1}, X_{2}, X_{3} \rrbracket$ (thus $X_{i} P_{i} X_{i}^{-1}=Q_{i}$ ). Consider the following cases.

(a) Assume $g \in H_{0}\left(q^{n}\right)$ and $P, Q \leq H_{0}\left(q^{n}\right)$. Since $\varphi_{u}$ preserves fusion in $S L_{2}\left(q^{n}\right)$, there is $Y_{3} \in S L_{2}\left(q^{n}\right)$ such that $Y_{3} \varphi_{u}\left(P_{3}\right) Y_{3}^{-1}=\varphi_{u}\left(Q_{3}\right)$ and $\left.c_{Y_{3}} \circ \varphi_{u}\right|_{P_{3}}=\left.\varphi_{u} \circ c_{X_{3}}\right|_{P_{3}}$. Then $h \stackrel{\text { def }}{=} \llbracket X_{1}, X_{2}, Y_{3} \rrbracket$ satisfies (1).

(b) Assume $g \notin H_{0}\left(q^{n}\right)$ and $P, Q \leq H_{0}\left(q^{n}\right)$. Write $g=g^{\prime} \cdot \llbracket Y, Y, Y \rrbracket$, where $g^{\prime} \in H_{0}\left(q^{n}\right)$. Choose $h^{\prime}$ as in (a), so that (1) is satisfied with $g, h$ replaced by $g^{\prime}, h^{\prime}$. Then the element $h=h^{\prime} \cdot \llbracket Y, Y, Y^{u} \rrbracket$ satisfies (1).

(c) Finally, assume that $P, Q \not H_{0}\left(q^{n}\right)$. Then none of the subgroups $P_{i}, Q_{i} \leq$ $S L_{2}\left(q^{2 n}\right)$ is contained in $S L_{2}\left(q^{n}\right)$. By the same procedure as was used in (a), we can find $h \in H_{0}\left(q^{2 n}\right)$ which satisfies (1); the problem is to do this so that $h \in H\left(q^{n}\right)$.

As noted above, fusion in $S L_{2}\left(q^{2 n}\right)$ is generated by inner automorphisms of $Q\left(q^{2 n}\right)$ and automorphisms of subgroups isomorphic to $Q_{8}$. Hence if $P_{i}$ 
is not isomorphic to $Q_{8}$ or one of its subgroups, then there is $X_{i}^{\prime} \in Q\left(q^{2 n}\right)$ such that $\left.c_{X_{i}}\right|_{P_{i}}=\left.c_{X_{i}^{\prime}}\right|_{P_{i}}$. If, on the other hand, $P_{i}$ (and hence $Q_{i}$ ) is isomorphic to $Q_{8}$ or $C_{4}$, then $P_{i} \leq\left\langle A, Y^{r} B\right\rangle$ and $Q_{i} \leq\left\langle A, Y^{s} B\right\rangle$ for some odd $r, s \in \mathbb{Z}$, and we can choose $k \in \mathbb{Z}$ such that $X_{i}^{\prime} \stackrel{\text { def }}{=} Y^{k} \in Q\left(q^{2 n}\right)$ has the same conjugation action as $X_{i}$. Thus in all cases, we can write $X_{i}=X_{i}^{\prime} X_{i}^{\prime \prime}$ for some $X_{i}^{\prime} \in Q\left(q^{2 n}\right)$ and some $X_{i}^{\prime \prime} \in C_{S L_{2}\left(q^{2 n}\right) \cdot\langle Y\rangle}\left(P_{i}\right)$.

The subgroups of $Q\left(q^{2 n}\right)$ which are centralized by elements in the coset $S L_{2}\left(q^{n}\right) \cdot Y$ are precisely the cyclic subgroups. (The quaternion subgroups of order $\geq 8$ are all centric in $S L_{2}\left(q^{2 n}\right)$.) Hence we can choose elements $Y_{i}^{\prime \prime}$ as follows: $Y_{i}^{\prime \prime}=1$ if $X_{i}^{\prime \prime} \in S L_{2}\left(q^{n}\right)$, and $Y_{i}^{\prime \prime} \in S L_{2}\left(q^{n}\right) \cdot Y$ and centralizes $\varphi_{u}\left(P_{i}\right)$ if $X_{i}^{\prime \prime} \in S L_{2}\left(q^{n}\right) \cdot Y$. We now define

$$
h=\llbracket X_{1}^{\prime}, X_{2}^{\prime}, \varphi_{u}\left(X_{3}^{\prime}\right) \rrbracket \cdot \llbracket Y_{1}^{\prime \prime}, Y_{2}^{\prime \prime}, Y_{3}^{\prime \prime} \rrbracket ;
$$

then $h \in H\left(q^{n}\right)$ and satisfies (1).

This shows that $\widehat{\delta}_{u}$ preserves $H\left(q^{n}\right)$-fusion as an automorphism of $S_{0}\left(q^{n}\right)$. Also, $\widehat{\gamma}$ and $c_{\tau}$ preserve fusion, since both extend to automorphisms of $H\left(q^{n}\right)$; and hence $\widehat{\gamma}_{u}=\widehat{\delta}_{u} \widehat{\gamma} \widehat{\delta}_{u}^{-1}$ also preserves fusion.

Let $\psi=\psi^{q^{n}} \in \operatorname{Aut}\left(\operatorname{Spin}_{7}\left(q^{\infty}\right)\right)$ be induced by the field automorphism $x \mapsto$ $x^{q^{n}}$. By [2, Proposition A.9(a)], if $E \leq \operatorname{Spin}_{7}\left(q^{n}\right)$ is an arbitrary elementary abelian 2-subgroup of rank 4 , then there is an element $a \in \operatorname{Spin}_{7}\left(q^{\infty}\right)$ such that $a E a^{-1}=E_{*}$, and we define

$$
x_{\mathcal{C}}(E)=a^{-1} \psi(a) .
$$

Then $x_{\mathcal{C}}(E) \in E$, and is independent of the choice of $a$.

In the following lemma, we correct the statement and proof of points (b) and (e). The proof of (e) is affected by both the changes in the statement of (b) and those in the definition of $\Gamma_{n}$.

Lemma 1.8 2, Lemma 2.8] Fix $n \geq 1$, set $E_{*}=\left\langle z, z_{1}, \widehat{A}, \widehat{B}\right\rangle \leq S\left(q^{n}\right)$, and let $\mathcal{C}$ be the $\operatorname{Spin}_{7}\left(q^{n}\right)$-conjugacy class of $E_{*}$. Let $\mathcal{E}_{4}^{U}$ be the set of all elementary abelian subgroups $E \leq S\left(q^{n}\right)$ of rank 4 which contain $U=\left\langle z, z_{1}\right\rangle$. Fix a generator $X \in C\left(q^{n}\right)$ (the 2-power torsion in $C_{S L_{2}\left(q^{n}\right)}(A)$ ), and choose $Y \in C\left(q^{2 n}\right)$ such that $Y^{2}=X$. Then the following hold.

(a) $E_{*}$ has type $I$. 
(b) Each subgroup in $\mathcal{E}_{4}^{U}$ which contains $\widehat{A}$ is of the form

$$
\begin{aligned}
E_{i j k}=\left\langle z, z_{1}, \widehat{A}, \llbracket X^{i} B, X^{j} B, X^{k} B \rrbracket\right\rangle & \text { or } \\
E_{i j k}^{\prime} & =\left\langle z, z_{1}, \widehat{A}, \llbracket X^{i} Y B, X^{j} Y B, X^{k} Y B \rrbracket\right\rangle .
\end{aligned}
$$

Each subgroup in $\mathcal{E}_{4}^{U}$ is $H\left(q^{n}\right)$-conjugate to one of these subgroups $E_{i j k}$ or $E_{i j k}^{\prime}$ for some $i, j, k \in \mathbb{Z}$.

(c) $\quad x_{\mathcal{C}}\left(E_{i j k}\right)=\llbracket(-I)^{i},(-I)^{j},(-I)^{k} \rrbracket$ and $x_{\mathcal{C}}\left(E_{i j k}^{\prime}\right)=\llbracket(-I)^{i},(-I)^{j},(-I)^{k} \rrbracket \cdot \widehat{A}$.

(d) All of the subgroups $E_{i j k}^{\prime}$ have type II. The subgroup $E_{i j k}$ has type I if and only if $i \equiv j(\bmod 2)$, and lies in $\mathcal{C}$ (is conjugate to $E_{*}$ ) if and only if $i \equiv j \equiv k(\bmod 2)$. The subgroups $E_{000}, E_{001}$, and $E_{100}$ thus represent the three conjugacy classes of rank four elementary abelian subgroups of $\operatorname{Spin}_{7}\left(q^{n}\right)$ (and $\left.E_{*}=E_{000}\right)$.

(e) For any $\varphi \in \Gamma_{n} \leq \operatorname{Aut}\left(S_{0}\left(q^{n}\right)\right)$ and any $E \in \mathcal{E}_{4}^{U}, \varphi\left(x_{\mathcal{C}}(E)\right)=x_{\mathcal{C}}(\varphi(E))$.

Proof We prove only points (b) and (e) here, and refer to 2 for the proofs of the other points.

(b) Assume first that $\widehat{A} \in E$; ie, that $E \geq A_{1}=\left\langle z, z_{1}, \widehat{A}\right\rangle$. By definition, $S\left(q^{n}\right)$ is generated by $A\left(q^{n}\right)$, whose elements clearly centralize $A_{1}$; and elements $\llbracket B^{i}, B^{j}, B^{k} \rrbracket$ for $i, j, k \in\{0,1\}$. Since an element of this form centralizes $\widehat{A}$ only if $i=j=k$, this shows that

$$
E \leq C_{S\left(q^{n}\right)}\left(\left\langle z, z_{1}, \widehat{A}\right\rangle\right)=A\left(q^{n}\right) \cdot\langle\widehat{B}\rangle .
$$

Since $A\left(q^{n}\right)$ is a finite abelian 2-group of rank 3 , we have $E=\left\langle z, z_{1}, \widehat{A}, g \widehat{B}\right\rangle$ for some $g \in A\left(q^{n}\right)$. Also,

$$
A\left(q^{n}\right)=\omega\left(C\left(q^{\infty}\right)^{3}\right) \cap \operatorname{Spin}_{7}\left(q^{n}\right)=\left\{\llbracket X^{i}, X^{j}, X^{k} \rrbracket, \llbracket X^{i} Y, X^{j} Y, X^{k} Y \rrbracket \mid i, j, k \in \mathbb{Z}\right\},
$$

and hence $E=E_{i j k}$ or $E_{i j k}^{\prime}$ for some $i, j, k \in \mathbb{Z}$.

Now let $E \in \mathcal{E}_{4}^{U}$ be arbitrary. Each element of $E$ has the form $\llbracket X_{1}, X_{2}, X_{3} \rrbracket$, where either $X_{i} \in S L_{2}\left(q^{n}\right)$ for all $i$, or $X_{i} \in S L_{2}\left(q^{n}\right) \cdot Y$ for all $i$ - and the elements of the first type $\left(X_{i} \in S L_{2}\left(q^{n}\right)\right)$ form a subgroup of index at most 2 . Since $U$ has index 4 in $E$, this means that there is some $g=\llbracket X_{1}, X_{2}, X_{3} \rrbracket \in$ $E \backslash U$ for which $X_{i} \in S L_{2}\left(q^{n}\right)$ for all $i$. Also, $\left|X_{i}\right|=4$ for all $i$, since $g \notin$ $U=\{\llbracket \pm I, \pm I, \pm I \rrbracket\}$. Since all elements of order 4 in $S L_{2}\left(q^{n}\right)$ are conjugate (cf. 44 3.6.23]), this implies that $g$ is $H\left(q^{n}\right)$-conjugate to $\widehat{A}$, and hence that $E$ is $H\left(q^{n}\right)$-conjugate to one of the above subgroups $E_{i j k}$ or $E_{i j k}^{\prime}$. 
(e) By construction, $x_{\mathcal{C}}(-)$ is preserved under conjugation by elements of the group $\operatorname{Spin}_{7}\left(q^{n}\right)$. Since $\Gamma_{n}$ is generated by $\widehat{\gamma}_{u}$ and conjugation by elements of $\operatorname{Spin}_{7}\left(q^{n}\right)$, it suffices to prove the result when $\varphi=\widehat{\gamma}_{u}$. Since $u \equiv 1(\bmod 4)$ by Proposition [1.5] $\widehat{\gamma}_{u}(\widehat{A})=\widehat{A}, \widehat{\gamma}_{u}\left(E_{i j k}\right)=E_{k^{\prime}, i^{\prime}, j^{\prime}}$, and $\widehat{\gamma}_{u}\left(E_{i j k}^{\prime}\right)=E_{k^{\prime \prime}, i^{\prime \prime}, j^{\prime \prime}}^{\prime}$ for some $i^{\prime} \equiv i^{\prime \prime} \equiv i(\bmod 2)$, and similarly for the other indices. Hence by (c), $\widehat{\gamma}_{u}\left(x_{\mathcal{C}}(E)\right)=x_{\mathcal{C}}\left(\widehat{\gamma}_{u}(E)\right)$ whenever $E=E_{i j k}$ or $E=E_{i j k}^{\prime}$ for some $i, j, k \in \mathbb{Z}$.

Now assume $E \in \mathcal{E}_{4}^{U}$ is not one of the subgroups $E_{i j k}$ or $E_{i j k}^{\prime}$. By (b), there is $g \in H\left(q^{n}\right)$ such that $E^{\prime} \stackrel{\text { def }}{=} g E g^{-1}$ is of this form. Since $\widehat{\gamma}_{u}$ preserves $H\left(q^{n}\right)$-fusion by Lemma 1.7 there is $h \in H\left(q^{n}\right)$ such that the following square commutes:

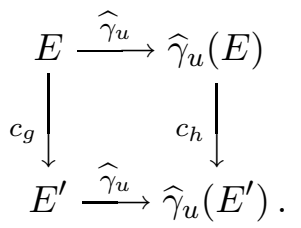

We have seen that $\widehat{\gamma}_{u}\left(x_{\mathcal{C}}\left(E^{\prime}\right)\right)=x_{\mathcal{C}}\left(\widehat{\gamma}_{u}\left(E^{\prime}\right)\right)$; and also that $c_{g}$ and $c_{h}$ preserve $x_{\mathcal{C}}(-)$. Hence $\widehat{\gamma}_{u}\left(x_{\mathcal{C}}(E)\right)=x_{\mathcal{C}}\left(\widehat{\gamma}_{u}(E)\right)$ by the commutativity of the square.

The following is the crucial result needed to apply Proposition 1.3. The statement is exactly the same as that in [2], but the proof has to be modified slightly due to the changed definition of $\Gamma_{n}$ (hence of $\mathcal{F}_{n}$ ).

Proposition 1.9 [2, Proposition 2.9] Fix $n \geq 1$. Let $E \leq S\left(q^{n}\right)$ be an elementary abelian subgroup of rank 3 which contains $U$, and such that $C_{S\left(q^{n}\right)}(E)$ $\in \operatorname{Syl}_{2}\left(C_{\operatorname{Spin}_{7}\left(q^{n}\right)}(E)\right)$. Then

$$
\left\{\varphi \in \operatorname{Aut}_{\mathcal{F}_{n}}\left(C_{S\left(q^{n}\right)}(E)\right) \mid \varphi(z)=z\right\}=\operatorname{Aut}_{\operatorname{Spin}_{7}\left(q^{n}\right)}\left(C_{S\left(q^{n}\right)}(E)\right) .
$$

\section{Proof Set}

$$
\text { Spin }=\operatorname{Spin}_{7}\left(q^{n}\right), \quad S=S\left(q^{n}\right), \quad \Gamma=\Gamma_{n}, \quad \text { and } \quad \mathcal{F}=\mathcal{F}_{n}
$$

for short, and consider the subgroups

$$
R_{0}=R_{0}\left(q^{n}\right) \stackrel{\text { def }}{=} A\left(q^{n}\right) \quad \text { and } \quad R_{1}=R_{1}\left(q^{n}\right) \stackrel{\text { def }}{=} C_{S}(\langle U, \widehat{A}\rangle)=\left\langle R_{0}, \widehat{B}\right\rangle .
$$

Then

$$
R_{0} \cong\left(C_{2^{k}}\right)^{3} \quad \text { and } \quad R_{1}=R_{0} \rtimes\langle\widehat{B}\rangle,
$$

where $2^{k}$ is the largest power which divides $q^{n} \pm 1$, and where $\widehat{B}=\llbracket B, B, B \rrbracket$ has order 2 and acts on $R_{0}$ via $\left(g \mapsto g^{-1}\right)$. Also,

$$
\langle U, \widehat{A}\rangle=\langle\llbracket \pm I, \pm I, \pm I \rrbracket, \llbracket A, A, A \rrbracket\rangle \cong C_{2}^{3}
$$


is the 2-torsion subgroup of $R_{0}$. It was shown in the proof of 2 , Proposition $2.9]$ that

$$
R_{0} \text { is the only subgroup of } S \text { isomorphic to }\left(C_{2^{k}}\right)^{3} \text {. }
$$

Let $E \leq S$ be an elementary abelian subgroup of rank 3 which contains $U$, and such that $C_{S}(E) \in \operatorname{Syl}_{2}\left(C_{\text {Spin }}(E)\right)$. There are two cases to consider: that where $E \leq R_{0}$ and that where $E \not \leq R_{0}$.

Case 1 Assume $E \leq R_{0}$. Since $R_{0}$ is abelian of rank 3 , we must have $E=\langle U, \widehat{A}\rangle$, the 2-torsion subgroup of $R_{0}$, and $C_{S}(E)=R_{1}$. Also, by (2), neither $R_{0}$ nor $R_{1}$ is isomorphic to any other subgroup of $S$; and hence

$$
\operatorname{Aut}_{\mathcal{F}}\left(R_{i}\right)=\left\langle\operatorname{Aut}_{\text {Spin }}\left(R_{i}\right), \operatorname{Aut}_{\Gamma}\left(R_{i}\right)\right\rangle=\left\langle\operatorname{Aut}_{\text {Spin }}\left(R_{i}\right),\left.\gamma_{u}\right|_{R_{i}}\right\rangle \quad \text { for } i=0,1 .
$$

(Recall that $\operatorname{Aut}_{\Gamma}\left(R_{i}\right)$ is generated by $\operatorname{Aut}_{S_{0}\left(q^{n}\right)}\left(R_{i}\right)$ and the restrictions of $c_{\tau}$ and $\left.\gamma_{u}.\right)$ Hence by Proposition 1.5(b),

$$
\operatorname{Aut}_{\mathcal{F}}\left(R_{0}\right)=\left\langle\operatorname{Aut}_{\text {Spin }}\left(R_{0}\right),\left.\gamma_{u}\right|_{R_{0}}\right\rangle \cong C_{2} \times G L_{3}(2) .
$$

In other words, if we let $A_{1}=\left\langle z, z_{1}, \widehat{A}\right\rangle$ denote the 2-torsion subgroup of $R_{0}$, then restriction to $A_{1}$ sends $\operatorname{Aut}_{\mathcal{F}}\left(R_{0}\right)$ onto $\operatorname{Aut}\left(A_{1}\right)$ with kernel $\left\langle c_{\widehat{B}}\right\rangle$ of order 2. Since $\operatorname{Aut}_{\text {Spin }}\left(A_{1}\right)$ is the group of all automorphisms of $A_{1}$ which send $z$ to itself [2, Proposition A.8], this shows that

$$
\operatorname{Aut}_{\text {Spin }}\left(R_{0}\right)=\left\{\varphi \in \operatorname{Aut}_{\mathcal{F}}\left(R_{0}\right) \mid \varphi(z)=z\right\} .
$$

In the proof of [2, Proposition 2.9] (see formula (5) in that proof), we show the first of the following two equalities:

$$
\begin{aligned}
\operatorname{Aut}_{\text {Spin }}\left(R_{1}\right) & =\operatorname{Inn}\left(R_{1}\right) \cdot\left\{\varphi \in \operatorname{Aut}_{\text {Spin }}\left(R_{1}\right) \mid \varphi(\widehat{B})=\widehat{B}\right\} \\
& =\operatorname{Inn}\left(R_{1}\right) \cdot\left\{\varphi \in \operatorname{Aut}\left(R_{1}\right)|\varphi(\widehat{B})=\widehat{B}, \varphi|_{R_{0}} \in \operatorname{Aut}_{\text {Spin }}\left(R_{0}\right)\right\}
\end{aligned}
$$

The second equality holds since $R_{1}=\left\langle R_{0}, \widehat{B}\right\rangle$, and since $R_{0}$ is the unique abelian subgroup of $R_{1}$ of index 2. Since $\widehat{\gamma}_{u}\left(R_{0}\right)=R_{0}$ and $\widehat{\gamma}_{u}(\widehat{B})=\widehat{B}$, this, together with (3), shows that

$$
\operatorname{Aut}_{\mathcal{F}}\left(R_{1}\right)=\operatorname{Inn}\left(R_{1}\right) \cdot\left\{\varphi \in \operatorname{Aut}\left(R_{1}\right)|\varphi(\widehat{B})=\widehat{B}, \varphi|_{R_{0}} \in \operatorname{Aut}_{\mathcal{F}}\left(R_{0}\right)\right\},
$$

and hence that

$$
\begin{aligned}
\{\varphi \in & \left.\operatorname{Aut}_{\mathcal{F}}\left(R_{1}\right) \mid \varphi(z)=z\right\} \\
& =\operatorname{Inn}\left(R_{1}\right) \cdot\left\{\varphi \in \operatorname{Aut}\left(R_{1}\right)|\varphi(\widehat{B})=\widehat{B}, \varphi(z)=z, \varphi|_{R_{0}} \in \operatorname{Aut}_{\mathcal{F}}\left(R_{0}\right)\right\} \\
& =\operatorname{Inn}\left(R_{1}\right) \cdot\left\{\varphi \in \operatorname{Aut}\left(R_{1}\right)|\varphi(\widehat{B})=\widehat{B}, \varphi|_{R_{0}} \in \operatorname{Aut}_{\text {Spin }}\left(R_{0}\right)\right\}
\end{aligned}
$$


where the second equality follows from (4). If $\varphi \in \operatorname{Aut}\left(R_{1}\right)$ is such that $\varphi(\widehat{B})=\widehat{B}$ and $\left.\varphi\right|_{R_{0}}=\left.c_{x}\right|_{R_{0}}$ for some $x \in N_{\text {Spin }}\left(R_{0}\right)$, then $x$ normalizes $R_{1}$ (the centralizer of the 2-torsion in $\left.R_{0}\right), c_{x}(\widehat{B})=c_{y}(\widehat{B})$ for some $y \in R_{1}=$ $R_{0} \cdot\langle\widehat{B}\rangle$ by (5), so we can assume $y \in R_{0}$. Since $R_{0}=A\left(q^{\infty}\right)$ is abelian (so $\left[y, R_{0}\right]=1$ ), this implies $\left.c_{y^{-1} x}\right|_{R_{1}}=\varphi$, and hence $\varphi \in \operatorname{Aut}_{\operatorname{Spin}}\left(R_{1}\right)$. So $\left\{\varphi \in \operatorname{Aut}_{\mathcal{F}}\left(R_{1}\right) \mid \varphi(z)=z\right\} \leq \operatorname{Aut}_{\text {Spin }}\left(R_{1}\right)$ by $(6)$, and the opposite inclusion is clear.

Case 2 Now assume that $E \not \leq R_{0}$. By assumption, $U \leq E$ (hence $E \leq$ $\left.C_{S}(E) \leq C_{S}(U)\right)$, and $C_{S}(E)$ is a Sylow subgroup of $C_{\text {Spin }}(E)$. Also, $E$ contains an element of the form $g \cdot \llbracket B^{i}, B^{j}, B^{k} \rrbracket$ for $g \in R_{0}=A\left(q^{n}\right)$ and some $i, j, k$ not all even, and hence $A\left(q^{n}\right) \not \leq C_{S}(E)$. Hence by $(2), C_{S}(E)$ is not isomorphic to $R_{1}=C_{S}\left(\left\langle z, z_{1}, \widehat{A}\right\rangle\right)$, and this shows that $E$ is not Spin-conjugate to $\left\langle z, z_{1}, \widehat{A}\right\rangle$. By [2, Proposition A.8], Spin contains exactly two conjugacy classes of rank 3 subgroups containing $z$, and thus $E$ must have type II. So by 2. Proposition A.8(d)], $C_{S}(E)$ is elementary abelian of rank 4, and also has type II.

Let $\mathcal{C}$ be the $\operatorname{Spin}_{7}\left(q^{n}\right)$-conjugacy class of the subgroup $E_{*}=\langle U, \widehat{A}, \widehat{B}\rangle \cong C_{2}^{4}$, which by Lemma 1.8(a) has type I. Let $\mathcal{E}^{\prime}$ be the set of all subgroups of $S$ which are elementary abelian of rank 4 , contain $U$, and are not in $\mathcal{C}$. By Lemma 1.8(e), for any $\varphi \in \operatorname{Iso}_{\Gamma}\left(E^{\prime}, E^{\prime \prime}\right)$ and any $E^{\prime} \in \mathcal{E}^{\prime}, E^{\prime \prime} \stackrel{\text { def }}{=} \varphi\left(E^{\prime}\right) \in \mathcal{E}^{\prime}$, and $\varphi$ sends $x_{\mathcal{C}}\left(E^{\prime}\right)$ to $x_{\mathcal{C}}\left(E^{\prime \prime}\right)$. The same holds for $\varphi \in \operatorname{Isospin}_{S}\left(E^{\prime}, E^{\prime \prime}\right)$ by definition of the elements $x_{\mathcal{C}}(-)$ ([2, Proposition A.9]). Since $C_{S}(E) \in \mathcal{E}^{\prime}$, this shows that all elements of $\operatorname{Aut}_{\mathcal{F}}\left(C_{S}(E)\right)$ send the element $x_{\mathcal{C}}\left(C_{S}(E)\right)$ to itself. By 2, Proposition A.9(c)], Aut $\operatorname{Spin}_{S}\left(C_{S}(E)\right)$ is the group of automorphisms which are the identity on the rank two subgroup $\left\langle x_{\mathcal{C}}\left(C_{S}(E)\right), z\right\rangle$; and (1) now follows.

The proof of the following lemma is essentially unchanged.

Lemma 1.10 2, Lemma 2.10] Fix $n \geq 1$, and let $E, E^{\prime} \leq S\left(q^{n}\right)$ be two elementary abelian subgroups of rank three which contain $U$, and which are $\Gamma_{n}$-conjugate. Then $E$ and $E^{\prime}$ are $\operatorname{Spin}_{7}\left(q^{n}\right)$-conjugate.

Proof Consider the sets

$$
\mathcal{J}_{1}=\left\{X \in S L_{2}\left(q^{n}\right) \mid X^{2}=-I\right\}
$$

and

$$
\mathcal{J}_{2}=\left\{X \in S L_{2}\left(q^{2 n}\right) \mid \psi^{q^{n}}(X)=-X, X^{2}=-I\right\} .
$$


Here, as usual, $\psi^{q^{n}}$ is induced by the field automorphism $\left(x \mapsto x^{q^{n}}\right)$. It was shown in the proof of [2, Lemma 2.10] that all elements in each of these sets are $S L_{2}(q)$-conjugate to each other.

Since $E$ and $E$ contain $U, E, E^{\prime} \leq C_{\operatorname{Spin}_{7}\left(q^{n}\right)}(U)$. By [2, Proposition 2.5(a)],

$$
C_{\operatorname{Spin}_{7}\left(q^{n}\right)}(U)=H\left(q^{n}\right) \stackrel{\text { def }}{=} \omega\left(S L_{2}\left(q^{\infty}\right)^{3}\right) \cap \operatorname{Spin}_{7}\left(q^{n}\right) .
$$

Thus

$$
E=\left\langle z, z_{1}, \llbracket X_{1}, X_{2}, X_{3} \rrbracket\right\rangle \quad \text { and } \quad E^{\prime}=\left\langle z, z_{1}, \llbracket X_{1}^{\prime}, X_{2}^{\prime}, X_{3}^{\prime} \rrbracket\right\rangle,
$$

where the $X_{i}$ are all in $\mathcal{J}_{1}$ or all in $\mathcal{J}_{2}$, and similarly for the $X_{i}^{\prime}$. Also, $E$ and $E^{\prime}$ are $\Gamma_{n}$-conjugate, and each element of $\Gamma_{n}$ leaves $U=\left\langle z, z_{1}\right\rangle$ and $\omega\left(S L_{2}\left(q^{n}\right)^{3}\right)$ invariant. Hence either $E$ and $E^{\prime}$ are both contained in $\omega\left(S L_{2}\left(q^{n}\right)^{3}\right)$, in which case the $X_{i}$ and $X_{i}^{\prime}$ are all in $\mathcal{J}_{1}$; or neither is contained in $\omega\left(S L_{2}\left(q^{n}\right)^{3}\right)$, in which case the $X_{i}$ and $X_{i}^{\prime}$ are all in $\mathcal{J}_{2}$. This shows that the $X_{i}$ and $X_{i}^{\prime}$ are all $S L_{2}\left(q^{n}\right)$-conjugate, and so $E$ and $E^{\prime}$ are $\operatorname{Spin}_{7}\left(q^{n}\right)$-conjugate.

We are now ready to prove:

Proposition 1.11 [2, Proposition 2.11] For a fixed odd prime power $q$, let $S\left(q^{n}\right) \leq S\left(q^{\infty}\right) \leq \operatorname{Spin}_{7}\left(q^{\infty}\right)$ be as defined above. Let $z \in Z\left(\operatorname{Spin}_{7}\left(q^{\infty}\right)\right)$ be the central element of order 2. Then for each $n, \mathcal{F}_{n}=\mathcal{F}_{\text {Sol }}\left(q^{n}\right)$ is saturated as a fusion system over $S\left(q^{n}\right)$, and satisfies the following conditions:

(a) For all $P, Q \leq S\left(q^{n}\right)$ which contain $z$, if $\alpha \in \operatorname{Hom}(P, Q)$ is such that $\alpha(z)=z$, then $\alpha \in \operatorname{Hom}_{\mathcal{F}_{n}}(P, Q)$ if and only if $\alpha \in \operatorname{Hom}_{\operatorname{Spin}_{7}\left(q^{n}\right)}(P, Q)$.

(b) $C_{\mathcal{F}_{n}}(z)=\mathcal{F}_{S\left(q^{n}\right)}\left(\operatorname{Spin}_{7}\left(q^{n}\right)\right)$ as fusion systems over $S\left(q^{n}\right)$.

(c) All involutions of $S\left(q^{n}\right)$ are $\mathcal{F}_{n}$-conjugate.

Furthermore, $\mathcal{F}_{m} \subseteq \mathcal{F}_{n}$ for $m \mid n$. The union of the $\mathcal{F}_{n}$ is thus a category $\mathcal{F}_{\text {Sol }}\left(q^{\infty}\right)$ whose objects are the finite subgroups of $S\left(q^{\infty}\right)$.

Proof We apply Proposition 1.3, where $p=2, G=\operatorname{Spin}_{7}\left(q^{n}\right), S=S\left(q^{n}\right)$, $Z=\langle z\rangle=Z(G), U=\left\langle z, z_{1}\right\rangle, C_{G}(U)=H\left(q^{n}\right), S_{0}=C_{S}(U)=S_{0}\left(q^{n}\right)$, and $\Gamma=\Gamma_{n} \leq \operatorname{Aut}\left(S_{0}\right)$. By Lemma 1.7 $\widehat{\gamma}_{u}$ preserves $H\left(q^{n}\right)$-fusion in $S_{0}$. Since $\Gamma$ is generated by $\widehat{\gamma}_{u}$ and certain automorphisms of $H\left(q^{n}\right)$, this shows that all automorphisms in $\Gamma$ preserve $H\left(q^{n}\right)$-fusion. 
Condition (a) in Proposition 1.3 (all noncentral involutions in $G$ are conjugate) holds since all subgroups in $\mathcal{E}_{2}$ are conjugate ([2, Proposition A.8]), and condition (b) holds by definition of $\Gamma$. Condition (c) holds since

$$
\{\gamma \in \Gamma \mid \gamma(z)=z\}=\operatorname{Inn}\left(S_{0}\left(q^{n}\right)\right) \cdot\left\langle c_{\tau}\right\rangle=\operatorname{Aut}_{N_{G}(U)}\left(S_{0}\left(q^{n}\right)\right)
$$

by [2, Proposition 2.5(b)]. Condition (d) was shown in Proposition [1.9, and condition (e) in Lemma 1.10. So by Proposition 1.3. $\mathcal{F}_{n}$ is a saturated fusion system, and $C_{\mathcal{F}_{n}}(Z)=\mathcal{F}_{S\left(q^{n}\right)}\left(\operatorname{Spin}_{7}\left(q^{n}\right)\right)$.

The proofs of the other statements remain unchanged.

In Section 3 of [2], these corrections affect only the proof of Lemma 3.1. In that proof, the groups $E_{100}$ and $E_{001}$ are not $\Gamma_{1}$-conjugate under the new definitions; instead $E_{100}$ is $\Gamma_{1}$-conjugate to a subgroup which is $\operatorname{Spin}_{7}(q)$ conjugate to $E_{001}$ (and hence the two are $\mathcal{F}$-conjugate). Also, when showing that $\operatorname{Aut}_{\mathcal{F}}\left(E_{001}\right)$ is the group of all automorphisms which fix $z=x_{\mathcal{C}}\left(E_{001}\right)$, it is important to know that all $\Gamma_{1}$-isomorphisms between subgroups in that conjugacy class preserve the elements $x_{\mathcal{C}}(-)$ (as shown in Lemma 1.8(e)), and not just that $\Gamma_{1}$-automorphisms of $E_{001}$ do so.

The changes do not affect the later sections. Just note that we are able to consider $\mathcal{F}_{\text {Sol }}\left(q^{n}\right)$ as a subcategory of $\mathcal{F}_{\text {Sol }}\left(q^{k n}\right)$ for $k>1$, because they were both chosen using the same "correction factor" $u \in\left(\widehat{\mathbb{Z}}_{2}\right)^{*}$ in the definitions of $\Gamma_{n}$ and $\Gamma_{k n}$.

\section{Proof of Proposition 1.3}

Proposition 1.3 follows from Lemmas 1.3, 1.4, and 1.5 in 2], once they are restated to assume the hypotheses of this new proposition, and not those of [2, Proposition 1.2]. The only one of these lemmas whose proof is affected by the change in hypotheses is Lemma 1.4, and so we restate and reprove it here.

Lemma 2.1 Assume the hypotheses of Proposition 1.3, and let

$$
\mathcal{F}=\left\langle\mathcal{F}_{S}(G) ; \mathcal{F}_{S_{0}}(\Gamma)\right\rangle
$$

be the fusion system generated by $G$ and $\Gamma$. Then for all $P, P^{\prime} \leq S$ which contain $Z$,

$$
\left\{\varphi \in \operatorname{Hom}_{\mathcal{F}}\left(P, P^{\prime}\right) \mid \varphi(Z)=Z\right\}=\operatorname{Hom}_{G}\left(P, P^{\prime}\right) .
$$


Proof Upon replacing $P^{\prime}$ by $\varphi(P) \leq P^{\prime}$, we can assume that $\varphi$ is an isomorphism, and thus that it factors as a composite of isomorphisms

$$
P=P_{0} \stackrel{\varphi_{1}}{\cong} P_{1} \stackrel{\varphi_{2}}{\cong} P_{2} \underset{\cong}{\stackrel{\varphi_{3}}{\cong}} \cdots \stackrel{\varphi_{k-1}}{\cong} P_{k-1} \underset{\cong}{\stackrel{\varphi_{k}}{\cong}} P_{k}=P^{\prime},
$$

where for each $i, \varphi_{i} \in \operatorname{Hom}_{G}\left(P_{i-1}, P_{i}\right)$ or $\varphi_{i} \in \operatorname{Hom}_{\Gamma}\left(P_{i-1}, P_{i}\right)$. Let $Z_{i} \leq Z\left(P_{i}\right)$ be the subgroups of order $p$ such that $Z_{0}=Z_{k}=Z$ and $Z_{i}=\varphi_{i}\left(Z_{i-1}\right)$.

To simplify the discussion, we say that a morphism in $\mathcal{F}$ is of type $(G)$ if it is given by conjugation by an element of $G$, and of type $(\Gamma)$ if it is the restriction of an automorphism in $\Gamma$. More generally, we say that a morphism is of type $(G, \Gamma)$ if it is the composite of a morphism of type $(G)$ followed by one of type $(\Gamma)$, etc. We regard $\operatorname{Id}_{P}$, for all $P \leq S$, to be of both types, even if $P \not \leq S_{0}$. By definition, if any nonidentity isomorphism is of type $(\Gamma)$, then its source and image are both contained in $S_{0}=C_{S}(U)$. In particular, if $P_{i} \not \leq S_{0}$ for any $0<i<k$, then $\varphi_{i}$ and $\varphi_{i+1}$ are both of type $(G)$, and we can remove it and replace the two morphisms by their composite. We can thus assume that $\left[P_{i}, U\right]=1$ for all $0<i<k$, and hence that $Z_{i} \leq Z\left(P_{i} U\right)$ for all $i$.

For each $i$, using [2, Lemma 1.3], choose some $\psi_{i} \in \operatorname{Hom}_{\mathcal{F}}\left(P_{i} U, S\right)$ such that $\psi_{i}\left(Z_{i}\right)=Z$. More precisely, using points (1) and (2) in 2. Lemma 1.3], we can choose $\psi_{i}$ to be of type $(\Gamma)$ if $Z_{i} \leq U$ (the inclusion if $Z_{i}=Z$ ), and to be of type $(G, \Gamma)$ if $Z_{i} \not \leq U$. Set $P_{i}^{\prime}=\psi_{i}\left(P_{i}\right)$. To keep track of the effect of morphisms on the subgroups $Z_{i}$, we write them as morphisms between pairs, as shown below. Thus, $\varphi$ factors as a composite of isomorphisms

$$
\left(P_{i-1}^{\prime}, Z\right) \stackrel{\psi_{i-1}^{-1}}{\longrightarrow}\left(P_{i-1}, Z_{i-1}\right) \stackrel{\varphi_{i}}{\longrightarrow}\left(P_{i}, Z_{i}\right) \stackrel{\psi_{i}}{\longrightarrow}\left(P_{i}^{\prime}, Z\right) .
$$

If $\varphi_{i}$ is of type $(G)$, then this composite (after replacing adjacent morphisms of the same type by their composite) is of type $(\Gamma, G, \Gamma)$. If $\varphi_{i}$ is of type $(\Gamma)$, then the composite is again of type $(\Gamma, G, \Gamma)$ if either $Z_{i-1} \leq U$ or $Z_{i} \leq U$, and is of type $(\Gamma, G, \Gamma, G, \Gamma)$ if neither $Z_{i-1}$ nor $Z_{i}$ is contained in $U$. So we are reduced to assuming that $\varphi$ is of one of these two forms.

Case 1 Assume first that $\varphi$ is of type $(\Gamma, G, \Gamma)$; ie, a composite of isomorphisms of the form

$$
\left(P_{0}, Z\right) \underset{(\Gamma)}{\stackrel{\varphi_{1}}{\longrightarrow}}\left(P_{1}, Z_{1}\right) \underset{(G)}{\stackrel{\varphi_{2}}{\longrightarrow}}\left(P_{2}, Z_{2}\right) \underset{(\Gamma)}{\stackrel{\varphi_{3}}{\longrightarrow}}\left(P_{3}, Z\right) .
$$

Then $Z_{1}=Z$ if and only if $Z_{2}=Z$ because $\varphi_{2}$ is of type $(G)$. If $Z_{1}=Z_{2}=Z$, then $\varphi_{1}$ and $\varphi_{3}$ are of type $(G)$ by Proposition [1.3(c), and the result follows.

If $Z_{1} \neq Z \neq Z_{2}$, then $U=Z Z_{1}=Z Z_{2}$, and thus $\varphi_{2}(U)=U$. Neither $\varphi_{1}$ nor $\varphi_{3}$ can be the identity, so $P_{i} \leq S_{0}=C_{S}(U)$ for all $i$ by definition of 
$\operatorname{Hom}_{\Gamma}(-,-)$. Let $\gamma_{1}, \gamma_{3} \in \Gamma$ be such that $\varphi_{i}=\left.\gamma_{i}\right|_{P_{i-1}}$, and let $g \in N_{G}(U)$ be such that $\varphi_{2}=c_{g}$. Then $g C_{S}(U) g^{-1} \in \operatorname{Syl}_{p}\left(C_{G}(U)\right)$, so there is $h \in C_{G}(U)$ such that $h g \in N\left(C_{S}(U)\right)$. Then $c_{h g} \in \Gamma$ by Proposition 1.3. (c).

Since $\gamma_{3}$ preserves $C_{G}(U)$-fusion among subgroups of $S_{0}$, there is $g^{\prime} \in C_{G}(U)$ such that $c_{g^{\prime}}=\gamma_{3} \circ c_{h}^{-1} \circ \gamma_{3}^{-1}$. Thus $\varphi$ is the composite

$$
P_{0} \underset{(\Gamma)}{\stackrel{\varphi_{1}}{\longrightarrow}} P_{1} \underset{(\Gamma)}{\stackrel{c_{h g}}{\longrightarrow}} h P_{2} h^{-1} \underset{(\Gamma)}{\stackrel{\gamma_{3}}{\longrightarrow}} \gamma_{3}\left(h P_{2} h^{-1}\right) \underset{(G)}{\stackrel{c_{g^{\prime}}}{\longrightarrow}} P_{3} .
$$

The composite of the first three isomorphisms is of type $(\Gamma)$ and sends $Z$ to $g^{\prime-1} Z g^{\prime}=Z$, hence is of type $(G)$ by Proposition 1.3(c) again, and so $\varphi$ is also of type $(G)$.

Case 2 Assume now that $\varphi$ is of type $(\Gamma, G, \Gamma, G, \Gamma)$; more precisely, that it is a composite of the form

$$
\begin{aligned}
\left(P_{0}, Z\right) \underset{(\Gamma)}{\stackrel{\varphi_{1}}{\longrightarrow}}\left(P_{1}, Z_{1}\right) \underset{(G)}{\stackrel{\varphi_{2}}{\longrightarrow}}\left(P_{2}, Z_{2}\right) \underset{(\Gamma)}{\stackrel{\varphi_{3}}{\longrightarrow}} & \left(P_{3}, Z_{3}\right) \\
& \stackrel{\varphi_{4}}{(G)}\left(P_{4}, Z_{4}\right) \stackrel{\varphi_{5}}{\underset{(\Gamma)}{\longrightarrow}}\left(P_{5}, Z\right),
\end{aligned}
$$

where $Z_{2}, Z_{3} \not \leq U$. Then $Z_{1}, Z_{4} \leq U$ and are distinct from $Z$, and the groups $P_{0}, P_{1}, P_{4}, P_{5}$ all contain $U$ since $\varphi_{1}$ and $\varphi_{5}$ (being of type $(\Gamma)$ ) leave $U$ invariant. In particular, $P_{2}$ and $P_{3}$ contain $Z$, since $P_{1}$ and $P_{4}$ do and $\varphi_{2}, \varphi_{4}$ are of type $(G)$. We can also assume that $U \leq P_{2}, P_{3}$, since otherwise $P_{2} \cap U=Z$ or $P_{3} \cap U=Z, \varphi_{3}(Z)=Z$, and hence $\varphi_{3}$ is of type $(G)$ by Proposition 1.3(c) again. Finally, we assume that $P_{2}, P_{3} \leq S_{0}=C_{S}(U)$, since otherwise $\varphi_{3}=\mathrm{Id}$.

Let $E_{i} \leq P_{i}$ be the rank three elementary abelian subgroups defined by the requirements that $E_{2}=U Z_{2}, E_{3}=U Z_{3}$, and $\varphi_{i}\left(E_{i-1}\right)=E_{i}$. In particular, $E_{i} \leq Z\left(P_{i}\right)$ for $i=2,3$ (since $Z_{i} \leq Z\left(P_{i}\right)$, and $U \leq Z\left(P_{i}\right)$ by the above remarks); and hence $E_{i} \leq Z\left(P_{i}\right)$ for all $i$. Also, $U=Z Z_{4} \leq \varphi_{4}\left(E_{3}\right)=E_{4}$ since $\varphi_{4}(Z)=Z$, and thus $U=\varphi_{5}(U) \leq E_{5}$. Via similar considerations for $E_{0}$ and $E_{1}$, we see that $U \leq E_{i}$ for all $i$.

Set $H=C_{G}(U)$ for short. Let $\mathcal{E}_{3}$ be the set of all elementary abelian subgroups $E \leq S$ of rank three which contain $U$, and let $\mathcal{E}_{3}^{*} \subseteq \mathcal{E}_{3}$ be the set of all $E \in \mathcal{E}_{3}$ such that $C_{S}(E) \in \operatorname{Syl}_{p}\left(C_{G}(E)\right)$. Then for all $E \in \mathcal{E}_{3}$,

$$
C_{S}(E)=C_{S_{0}}(E) \quad \text { and } \quad C_{G}(E)=C_{H}(E)
$$

since $E \geq U$ (and $S_{0}=C_{S}(U)$ ). Thus $\mathcal{E}_{3}^{*}$ is the set of all subgroups $E \in \mathcal{E}_{3}$ which are fully centralized in the fusion system $\mathcal{F}_{S_{0}}(H)$, and so each subgroup in $\mathcal{E}_{3}$ is $H$-conjugate to a subgroup in $\mathcal{E}_{3}^{*}$. 
Let $\gamma_{1}, \gamma_{3}, \gamma_{5} \in \Gamma$ be such that $\varphi_{i}=\left.\gamma_{i}\right|_{P_{i-1}}$ for odd $i$. Let $g_{2}, g_{4} \in G$ be such that $\varphi_{i}$ is conjugation by $g_{i}$ for $i=2,4$. We will construct a commutative diagram of the following form

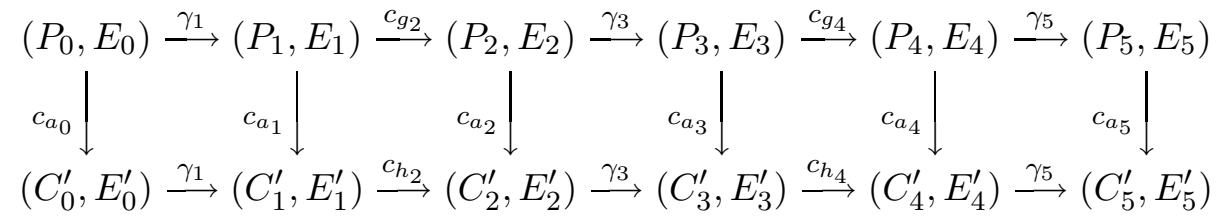

where $E_{i}^{\prime} \in \mathcal{E}_{3}^{*}, C_{i}^{\prime}=C_{S}\left(E_{i}^{\prime}\right), h_{2}, h_{4} \in G$, and $a_{i} \in H=C_{G}(U)$. To do this,

first choose $a_{0}^{\prime}, a_{2}^{\prime}, a_{4}^{\prime} \in H$ such that $E_{i}^{\prime} \stackrel{\text { def }}{=} a_{i}^{\prime} E_{i} a_{i}^{\prime-1} \in \mathcal{E}_{3}^{*}$ for $i=0,2,4$, and set $E_{i}^{\prime}=\gamma_{i}\left(E_{i-1}^{\prime}\right)$ for $i=1,3,5$. Then $C_{S}\left(E_{i}^{\prime}\right)=\gamma_{i}\left(C_{S}\left(E_{i-1}^{\prime}\right)\right)$ for $i=1,3,5$, so $C_{S}\left(E_{i}^{\prime}\right) \in \operatorname{Syl}_{p}\left(C_{H}\left(E_{i}^{\prime}\right)\right)$ and $E_{i}^{\prime} \in \mathcal{E}_{3}^{*}$ for all $i$. So we can choose $x_{0} \in C_{H}\left(E_{0}^{\prime}\right)$ such that $x_{0}\left(a_{0}^{\prime} P_{0} a_{0}^{\prime-1}\right) x_{0}^{-1} \leq C_{S}\left(E_{0}^{\prime}\right)$, and set $a_{0}=x_{0} a_{0}^{\prime} \in H$. Since $\gamma_{1} \in$ $\operatorname{Aut}\left(S_{0}\right)$ preserves $H$-fusion, there is $a_{1} \in H$ which makes the first square in the above diagram commute. Now choose $x_{2} \in C_{H}\left(E_{2}^{\prime}\right)$ such that

$$
x_{2}\left(\left(a_{2}^{\prime} g_{2} a_{1}^{-1}\right) C_{S}\left(E_{1}^{\prime}\right)\left(a_{2}^{\prime} g_{2} a_{1}^{-1}\right)^{-1}\right) x_{2}^{-1} \leq C_{S}\left(E_{2}^{\prime}\right),
$$

and set $a_{2}=x_{2} a_{2}^{\prime}$ and $h_{2}=a_{2} g_{2} a_{1}^{-1}$. Upon continuing this procedure we obtain the above diagram.

Let $\widehat{\varphi} \in \operatorname{Iso}_{\mathcal{F}}\left(C_{S}\left(E_{0}^{\prime}\right), C_{S}\left(E_{5}^{\prime}\right)\right)$ be the composite of the morphisms in the bottom row of the above diagram. Then $\widehat{\varphi}(Z)=Z$, since $\varphi$ and the $c_{a_{i}}$ all send $Z$ to itself. By Proposition [1.3(e), the rank three subgroups $E_{i}^{\prime}$ are all $G$-conjugate to each other. Choose $g \in G$ such that $g E_{5}^{\prime} g^{-1}=E_{0}^{\prime}$. Then $g \cdot C_{S}\left(E_{5}^{\prime}\right) \cdot g^{-1}$ and $C_{S}\left(E_{0}^{\prime}\right)$ are both Sylow $p$-subgroups of $C_{G}\left(E_{0}^{\prime}\right)$, so there is $h \in C_{G}\left(E_{0}^{\prime}\right)$ such that $(h g) C_{S}\left(E_{5}^{\prime}\right)(h g)^{-1}=C_{S}\left(E_{0}^{\prime}\right)$. By Proposition [1.3(d), $c_{h g} \circ \widehat{\varphi} \in \operatorname{Aut}_{\mathcal{F}}\left(C_{S}\left(E_{0}^{\prime}\right)\right)$ is of type $(G)$, so $\widehat{\varphi}$ is of type $(G)$, and hence $\varphi=c_{a_{5}}^{-1} \circ \widehat{\varphi} \circ c_{a_{0}}$ is also of type $(G)$.

\section{References}

[1] W Dwyer, C Wilkerson, A new finite loop space at the prime two, J. Amer. Math. Soc. 6 (1993) 37-64 MathReview

[2] R Levi, B Oliver, Construction of 2-local finite groups of a type studied by Solomon and Benson, Geom. Topol. 6 (2002) 917-990 MathReview

[3] R Solomon, Finite groups with Sylow 2-subgroups of type 3, J. Algebra 28 (1974) 182-198 MathReview

[4] M Suzuki, Group theory I, Grundlehren series 247, Springer-Verlag (1982) MathReview 